\title{
La vie humaine au rythme des saisons et des mois - Les Quatre âges de l'homme de Philippe de Novare et Les Douze mois figurez
}

\author{
Human life in the rhythm of the seasons and the months - \\ Philippe de Novare Les Quatre âges de l'homme \\ and Les Douze mois figurez
}

\author{
Anna Loba \\ Uniwersytet im. Adama Mickiewicza w Poznaniu \\ annaloba@amu.edu.pl
}

\begin{abstract}
The division of human life into ages is not only a sociological issue but also includes a philosophical, moral and religious reflection. The relationship between human life and the course of the year punctuated by the seasons and months has not remained without influence on the very notion of the ages of life. The question that arises is whether we can harmonize the unpredictable flow of human life with the calendar. The confrontation of two medieval works which develop this parallel: the treatise by Philippe de Novare Les Quatre âges de l'homme, and the anonymous poem Les Douze mois figurez, should provide an answer to this question.
\end{abstract}

Keywords: ages of man, calendar, age groups, time measurment

La division de la vie humaine en âges n'est pas seulement une question d'ordre sociologique car, comme le rappelle le philosophe Pierre-Henri Tavoillot, « les âges sont des catégories à la fois physiques et métaphysiques, à la fois profanes et $s a$ crées » (Tavoillot, 2010, p. 9). Concernant le sens global de la vie, ils répondent aux questions fondamentales : "pourquoi grandir ? ", " comment conduire sa vie ? ", " que signifie être adulte ? ». Et pourtant, il n'y a jamais eu une seule manière de diviser la vie de l'homme : les différentes classifications qui se sont succédées au 
cours des époques étaient associées à d'autres classifications, liées au fonctionnement du monde, de la nature et de l'histoire humaine (Schmitt, 2007, p. 815). La culture médiévale, tout en se réappropriant des modèles antiques, en a inventé des nouveaux pour cerner et définir les différentes périodes de la vie humaine. Pour les philosophes et les érudits mais aussi pour les médecins et les moralistes, les âges de la vie pouvaient être au nombre de trois, quatre, cinq, six, voire sept ou même douze.

L'indubitable correspondance entre la vie de l'homme et le cours de l'année rythmé par les saisons et les mois n'est pas restée sans influence sur la notion même des âges de la vie. Et pourtant, peut-on harmoniser le flux imprévisible de la vie humaine avec le calendrier? La confrontation de deux ouvrages médiévaux qui développent ce parallèle : le traité de Philippe de Novare, Les Quatre âges de l'homme et le poème anonyme Les Douze mois figurez, pourra, comme nous l'espérons, apporter une réponse à cette question.

Dans l'Antiquité, la vie humaine était généralement divisée en trois phases reflétant l'idée de croissance, de stabilité et de déclin. Ainsi, Aristote dans sa Rhétorique, distinguait trois âges de l'homme : la jeunesse, l'âge mûr et la vieillesse. Cette division d'ordre essentiellement biologique mettait l'accent sur la force qui portait tous les êtres vivants à croître, à se reproduire et à décliner, car, comme l'avait dit le philosophe : « il faut nécessairement que ce qui est une fois né croisse, se développe et périsse » (Aristote, 1846, p. 341). Dans cette conception, l'âge adulte était considéré comme une période intermédiaire : « Ceux qui sont dans la force de l'âge auront, évidemment, un caractère moral tenant le milieu entre les jeunes gens et les vieillards et retranchant ce qui est en excès chez les uns et les autres » (Aristote, 1883, p. 234).

C'est à Pythagore qu'on a attribué la division de la vie en quatre tranches égales de vingt ans chacune : " vingt ans pour l'enfance, vingt pour l'adolescence, vingt pour la jeunesse, autant pour la vieillesse » (Diogène de Laërte, 1847, p. 153). Selon la tradition, ce philosophe fut aussi le premier à établir une correspondance entre les quatre âges de l'homme et les quatre saisons de l'année. Ovide place dans ses $M e ́-$ tamorphoses l'enseignement de Pythagore sur la vie de l'homme divisée en quatre phases : « Ne voyez-vous pas l'année se présenter tour à tour sous quatre faces, image de la vie ? » (Ovide, 1969, p. 510). Le printemps est donc comparé à l'enfant au berceau, faible, délicat et nourri de lait. Quand la nature se ranime, les plantes se gonflent de sucs et se couvrent de fleurs, mais tout n'est encore qu'une promesse incertaine. L'été qui succède au printemps est l'âge de la jeunesse, la saison la plus vigoureuse, la plus ardente et la plus féconde. Puis vient l'automne, comparé à l'âge mûr, étape intermédiaire entre les ardeurs juvéniles et les glaces de la vieillesse. Enfin l'hiver arrive comme un vieillard tremblant et triste avec sa tête chauve ou couverte de cheveux blancs $^{1}$. Mais en fin de comptes, la théorie des quatre âges correspond à une triparti-

${ }^{1}$ La transposition en vers de ce passage des Métamorphoses par l'auteur anonyme de l'Ovide moralisé du début du XIVe siècle, commence ainsi : «Printemps, estez, autompne, yvers, / Qui ont diverses 
tion de la vie, la période du milieu, celle de la maturité, étant plus courte que les deux autres (Paravicini Bagliani, 1999, p. 10).

Au Moyen Âge, ces modèles antiques furent adaptés à une symbolique chrétienne qui tendait à intégrer la vie de l'homme dans l'histoire sainte. Même si la répartition en trois âges jouissait encore d'une certaine fortune, le chiffre trois pouvant être associé à la sainte Trinité, à la Sainte Famille ou aux Rois mages, d'autres durées imposaient d'autres correspondances. Saint Augustin et après lui Isidore de Séville, distinguent six âges de l'homme, mis en rapport avec six âges du monde. Le temps présent est donc assimilé à la vieillesse, car le monde court à sa perte : mundus senescit. Le système de sept âges de l'homme, en relation avec les sept planètes tournant autour de la terre, se prêtait à son tour aux parallèles avec les sept péchés capitaux, les sept vertus ou les sept arts libéraux. Shakespeare s'y réfère encore dans Comme il vous plaira où il fait prononcer à Jacques son fameux monologue : «Un homme, dans le cours de sa vie, joue différents rôles, et les actes de la pièce sont les sept âges » (Shakespeare, 1863, p. 252) 2 .

Malgré des réinterprétations chrétiennes, les schémas de la vie au Moyen Âge ont prolongé les conceptions anciennes. Selon Denis Hüe, il n'est pas surprenant de retrouver dans cette diversité des âges toute une série de nombres de forte valeur symbolique, « comme si, en rattachant cet écoulement de la vie humaine à un des nombres forts du cosmos, on le rapprochait davantage de l'ordre du monde » (Hüe, 1987). Parmi ces multiples divisions deux vont attirer notre attention particulière : celle en quatre et celle en douze âges de la vie, car elles sont liées au rythme des saisons de l'année et aux changements des mois. Sur ce plan, comme le rappelle Agostino Paravicini Bagliani, le schéma qui a rencontré le plus de succès proposait quatre âges : pueritia ou infantia, iuventus, maturitas, senectus. Cette division quaternaire rendait possible un rapprochement entre l'homme, la nature et la symbolique chrétienne, celle des quatre Évangiles, des quatre fleuves du paradis, des quatre vertus théologales (Paravicini Bagliani, 1999, p. 16). En harmonie avec les spéculations des Anciens sur la nature, elle était conciliable avec le récit de la Genèse, selon lequel Dieu avait créé les quatre saisons le quatrième jour de la Création ${ }^{3}$. Le schéma des quatre âges permettait donc d'établir des liens avec le fondement de l'anthropologie antique et médiévale selon laquelle l'homme est un cosmos en miniature : il fait partie de l'ordre voulu par Dieu. Perçu comme un microcosme, le corps humain reproduit à sa mesure le macrocosme et se compose, comme lui, des quatre éléments déterminés par un mélange de quali-

qualitez, / Sont les quatre diversitez / De l'an, qui resamblent et sivent / Les estas des homes qui vivent » (Ovide moralisé, 1938, p. 204-205).

${ }^{2}$ Un souvenir de cette division se retrouve peut-être aussi dans le fameux fragment des Essais de Michel de Montaigne : « Je peints le passage : non un passage d'aage en autre, ou, comme dict le peuple, de sept en sept ans, mais de jour en jour, de minute en minute» (Montaigne, 1962, p. 782).

${ }^{3}$ « Que des corps de lumière soient faits dans le firmament du ciel, afin qu'ils séparent le jour et la nuit, et qu'ils servent de signes pour marquer les temps et les saisons, les jours et les années » (Gn 1, 14). 
tés naturelles : le chaud, le froid, le sec et l'humide. Ce schéma d'explication micro et macrocosmique fonctionnait à différents niveaux ce qui permettait l'élaboration des correspondances entre l'homme, l'âge et les saisons. Comme le rappelle Marylin Nicoud, il était en relation étroite avec la théorie humorale sur laquelle se fondait la médecine médiévale : "L'enfant, qui a une complexion sanguine, est donc chaud et humide comme l'air et comme le printemps ; l'adolescent, chaud et sec, est colérique comme le feu et l'été. L'homme mûr est quant à lui, à l'instar de la terre et de l'automne, théoriquement froid et $\mathrm{sec}$, tandis que le vieillard ressemble à l'hiver et à l'eau, comme eux froid et humide » (Nicoud, 1998, p. 61). Tout le monde physique, l'homme compris, dépend de l'équilibre entre ces qualités : si la jeunesse se caractérise par la chaleur et l'humidité, le vieillissement est provoqué par la surabondance de froid et de sec (Schmitt, 2007, p. 815).

En revanche, la division en douze âges, bien que ce nombre fût chargé d'une riche symbolique dans la tradition chrétienne, ne connaît un véritable succès que vers la fin du Moyen Âge (Dal, 1980, p. 10-11). Toutefois, comme le rappelle Javier Docampo, l'une des premières formulations théoriques du parallélisme entre les douze mois de l'année et les étapes de la vie humaine a été donnée par Hildegarde de Bingen dans la seconde moitié du XII ${ }^{\mathrm{e}}$ siècle (Docampo, 2017, p. 209). Dans la quatrième vision du Liber divinorum operum simplicis hominis (Le Livre des æuvres divines), la savante abbesse fait référence aux saisons et aux mois, au cycle régulier de la nature dont l'homme lui-même porte l'empreinte :

Dieu a donc consigné dans l'homme toutes les créatures. Il a aussi reproduit en lui l'ordre des différents moments de l'année. L'été correspond à l'homme éveillé, l'hiver à l'homme qui dort. L'hiver renferme en lui ce que l'été profère dans la joie. Le sommeil réconforte le dormeur pour qu'il soit rapidement apte à certaines œuvres, quand ses énergies s'éveillent. Il a donc distingué en lui les mois, discernant les qualités et les vertus... (Hildegarde de Bingen, 1989, p. 115-116)

L'homme évolue avec le temps : à chaque mois correspond donc un âge de la vie, une humeur particulière, un organe défini et une vertu (Cf. Hildegarde de Bingen, 1882, col. 878-883).

La correspondance entre l'âge de l'homme et les saisons de l'année d'un côté, et les mois de l'année d'un autre, se prête facilement à des considérations morales et trouve sa transposition littéraire dans deux ouvrages qui vont nous intéresser particulièrement. Philippe de Novare dans son fameux traité Les Quatre âges de l'homme, rédigé vers 1265 , considère les âges de la vie : « anfance et jovant et moien aage et viellece » (Philippe de Novare, 1888, p. 2) en fonction du cycle des saisons. L'enfance est donc comparée au printemps, la jeunesse à l'été, l'âge moyen à l'automne et enfin la vieillesse à l'hiver. Comme, suivant la division pythagoricienne, chaque phase compte vingt ans, la vie humaine, conformément à la Bible ne dépasse pas 
quatre-vingts ans, car, à en croire le Psalmiste : « Les jours de tous nos ans ne vont ordinairement qu'à soixante et dix années : si les plus forts vivent jusqu'à quatre-vingts ans, le surplus n'est que peine et douleur » (Ps 89, 10). L'âge de soixante-dix ans représenterait donc la limite ultime de la vie humaine, tandis que l'âge de quatre-vingts ans relèverait plutôt de l'exception. L'adoption du schéma quartenaire est un leitmotiv qui structure l'ouvrage de Philippe de Novare. Quatre valeurs morales : « soffrance et servise, valor et honor » (Philippe de Novare, 1888, p. 116) doivent s'appliquer à chacun des quatre âges.

La comparaison aux saisons de l'année n'apparaît pas dès le début du traité, elle n'est expliquée que dans la seconde partie du livre, consacrée à la jeunesse :

Li jovenz est comparez et afigurez a l'esté, car ausis comme il a .IIII. tens en droit aage d'ome, ausis comme il a .IIII. tens et saisons en l'an. Li premiers est li printens de Pascor ; li secons est estez, li tiers est rewains, li quarz est yver. Li printemps de Pascour seursenble a enfance, et estez a jouvent, et rewains au moyen aage, et yvers a viellesce (Philippe de Novare, 1888, p. 42).

Il serait juste et convenable, précise aussitôt Philippe de Novare, de débattre de cette question pour démontrer comment et pourquoi chacun de quatre âges ressemble à une saison de l'année, mais puisque cette démonstration serait trop longue, l'auteur s'en abstient. Au lieu de développer le sujet, il passe directement au parallèle de la jeunesse et de l'été. Bien que le temps de jeunesse soit décrit comme une belle et agréable période de la vie : « car l'an i a plus de joie que en autre tens, et de cortoisie et de largesse et de pooir de cors et de vigor et de valor » (Philippe de Novare, 1888, p. 37), elle inspire à Philippe de Novare plus de méfiance que de faveur. S'il est vrai que les jeunes gens sont joyeux, sociables et attirants de nature et l'âge juvénile abonde en plaisirs, pour l'auteur du traité c'est un âge périlleux, « li plus perilleux de touz les.IIII. tenz d'aage d'ome et de fame » (Philippe de Novare, 1888, p. 21), à cause de l' « eschaufemenz » qui lui est propre. Il se caractérise donc par le penchant à la luxure et à la violence, par l'arrogance, l'excès de confiance en soi et l'insolence ${ }^{4}$. Mais grâce à leur chaleur naturelle, les jeunes gens sont forts et en bonne santé. C'est pourquoi ils doivent mettre à profit leur grande vigueur physique pour acquérir la fortune et assurer leur vieillesse. S'il est vrai que le travail permet aux jeunes de s'établir, la jeunesse n'est pas considérée pour autant comme le moment privilégié de la vie, au contraire, c'est l'âge de dépendance caractérisé par le « servise » envers Dieu, envers un seigneur ou un maître (Lorcin, 1994, p. 20). L'âge idéal c'est donc la maturité, « moien aage » qui va de quarante à soixante ans. C'est le meilleur temps de la vie où l'homme atteint l'équilibre et la connaissance de soi, il est en plein épanouissement de ses facultés, parfaitement dominé par la force de caractère, la

${ }^{4}$ « Il i a plusor josnes qui sont si outrecuidié qu'il cuident tout savoir et pooir et valoir » (Philippe de Novare, 1888, p. 22). 
sagesse et la bonté. La vieillesse, dont Philippe de Novare dit qu'elle « vaut pis que yvers a toute sa froidure » (Philippe de Novare, 1888, p. 44), malgré tous ses maux et douleurs ne fait vraiment peur qu'à ceux qui n'ayant pas assez travaillé, n'ont pas amassé les biens dans leur jeunesse. Tout ce qu'on récolte en été servira à assurer les provisions d'hiver. Ainsi l'homme doit imiter la fourmi prévoyante qui amasse des grains pour la morte saison : " Nès li fremiz porchace son vivre en esté, et met le grain en son pertuis por avoir en yver » (Philippe de Novare, 1888, p. 43). Les deux étapes antérieures, enfance et jeunesse sont donc considérées comme des étapes préparatoires à la maturité.

Si la jeunesse, malgré ses charmes, n'est pas montrée comme une période pleinement heureuse de la vie humaine, l'enfance paraît un moment encore plus problématique. Le petit enfant est perçu comme un être inaccompli et irraisonnable qui exige des soins et une attention constants. Selon Philippe de Novare, l'amour naturel des parents est nécessaire pour supporter ces êtres malpropres et criards, car, dit-il : « il sont si ort et si annieus en petitesce, et si mal et si divers quant il sont .I. po grandet, que a painnes en norriroit on nul » (Philippe de Novare, 1888, p. 2-3). Pour Jean Batany, c'est le mot « divers » qui est le terme-clé de ce passage : « le personnage divers, c'est celui dont on ne peut prévoir les actes, en qui on ne peut avoir confiance - une cause d'angoisse perpétuelle pour une époque toujours en quête de sécurité matérielle et morale. Voilà sans doute la raison la plus profonde de la méfiance du Moyen Âge à l'égard de l'enfant » (Batany, 1973, p. 125).

La comparaison entre les quatre âges et les saisons, telle qu'elle émerge du traité de Philippe de Novare peut paraître décevante voire tout à fait plate : « assez joliment écrite pour qu'on en oublie la banalité » (Grossel, 2016, p. 35). En effet, les allusions météorologiques, la description de la nature, les caractéristiques de la spécificité de chaque saison de l'année sont absentes au profit de l'évocation des travaux des champs bien connus des calendriers de pierre sculptés sur les portails des cathédrales (Mâle, 1910, p. 85-96). L'enfance, associée au printemps fait penser à la période de croissance de la végétation. Dans ce sens, les soins apportés par les parents, les nourrices et les éducateurs visent d'abord à veiller au développement de leur progéniture qui pousse et grandit telle une jeune plante fragile. La longue période de l'enfance qui dure jusqu'à vingt ans correspond à l'idée du printemps avec ses espoirs et ses inquiétudes, à cette saison de l'année où il faut semer, planter et soigner ses plantations. Dans cette optique, les efforts des parents et des éducateurs sont comparables aux travaux des jardiniers et des agriculteurs : " chascuns voit et seit que le blef que l'an semme et les aubres que l'an plante, tant comme il sont petit, il ont mestier de garde et de norriture, d'aigue et de labor » (Philippe de Novare, 1888, p. 68). La jeunesse est assimilée à l'été où il faut profiter de la longueur des jours pour travailler : faucher le blé, le battre et vanner, le rentrer dans la grange pour avoir de quoi manger pendant toute l'année. On cueille les fruits de la terre et on les ramasse, « porveamment a droite saison », car, comme précise l'auteur du traité : « quant l'an atant trop, li espiz dou blé ploie vers terre; et li grains seche 
et chiet des aubres ; soiche la fueille et chiet li fruiz » (Philippe de Novare, 1888, p. 69). L'âge mûr, associé à l'automne, est le temps où l'on peut profiter du fruit de ses efforts : « Ou tens dou moien aage est li fruiz meürs : ce est la quenoissance et le pooir de nature ; et adonc doivent fructefier et trier le bien dou mal» (Philippe de Novare, 1888 , p. 69). La vieillesse comparée à l'hiver est la période de la décrépitude du corps humain et de la dégénérescence de la végétation : les membres de l'homme se courbent et sa tête ploie à l'image d'un arbre qui a perdu ses feuilles et penche vers la terre. "Car de terre sont, et en terre revendront», conclut Philippe de Novare en pensant à la mort des hommes et à la léthargie de la nature (Philippe de Novare, 1888, p. 69).

Dans l'imaginaire des âges de la vie, le passage des saisons aux mois s'opère relativement tard. Vers la fin du Moyen Âge, la popularité du schéma des « douze âges » est attestée par toute une famille de poèmes cherchant à établir des similitudes entre les caractéristiques des mois et les étapes de la vie humaine (Dal, 1980, p. 10-20). La source la plus ancienne de ce motif littéraire est un poème anonyme du XIV siècle, Les Douze mois figurez, édité par Joseph Morawski (Morawski, 1926, p. 351-363). Selon Glynnis M. Cropp, il est facile de comprendre l'extension du parallèle des quatre saisons à celui des douze mois, car à l'époque de la rédaction du poème l'iconographie du calendrier peint et sculpté était déjà bien établie et parfaitement bien connue (Cropp, 1980, p. 262-263). La vie de l'homme, divisée en douze tranches de six ans chacune, se termine à soixante-douze ans, ce qui donne un laps de temps plus court par rapport à la durée prévue par Philippe de Novare. Mais si les âges de l'homme doivent être comparés aux mois, le choix de multiplications est limité. Lorsqu'on assigne à chaque phase la durée de cinq ans, l'homme doit mourir à soixante ans, ce qui semble trop tôt, même si c'est l'âge prôné par Eustache Deschamps dans Double lay de la fragilité humaine, adaptation française du célébrissime traité De miseria humanae conditionis de Lothaire de Segni : «A bien vous amesurez, / Que .LX. ans ne durez, / - Pou passent oultre le sueil » (Eustache Deschamps, 1878, p. 264)5. Tandis que si on divise la vie humaine en douze périodes de sept ans, l'âge de l'homme dépasse le nombre de quatre-vingts ans, appuyé par l'autorité de la Bible.

Dans le poème des Douze mois figurez, l'homme change donc douze fois pendant sa vie, tout comme les mois changent selon la nature. Les six premières années de la vie humaine sont comparables à janvier : l'homme n'a ni force, ni capacités, ni connaissances, l'enfant «n'a ne force ne vertu » (Morawski, 1926, p. 356). Pendant les six années suivantes, il grandit et acquiert des connaissances, semblable au mois de février qui, vers sa fin, s'approche du printemps. À dix-huit ans, l'homme se réveille comme la nature en mars quand le temps commence à se faire beau et chaud, sa confiance en soi se développe : il « cuide valoir mille mars » (Morawski, 1926, p. 356).

${ }^{5}$ La version latine donnée par Eustache Deschamps est encore plus sévère : peu d'hommes atteignent quarante et très peu soixante ans («pauci nunc ad .XL., paucissimi ad .LX. annos perveniunt »). Cf. Eustache Deschamps, 1878, p. 265. 
Le mois d'avril, considéré au Moyen Âge comme le plus beau mois de l'année, signifie l'avènement du printemps et la renaissance de la nature : « l'erbe croist, l'arbre florist, / Les oyseaux reprennent leurs chans » (Morawski, 1926, p. 357). Le jeune homme qui atteint vingt-quatre ans devient fort, vertueux, beau, noble et amoureux. Lorsque la nature s'épanouit au mois de mai, « [1]e plus puissant des .XII. moys » (Morawski, 1926, p. 357), l'homme arrive à trente ans. Parvenu au sommet de son développement physique, fort et bien entraîné, il est apte à porter une épée. Le mois de juin, « de grant chaleur plains » (Morawski, 1926, p. 357), marque le solstice de la vie humaine : à trente-six ans l'homme commence à devenir raisonnable et avisé. Juillet est un mois où tout commence à décliner : " se commence a passer / La beauté d'une creature " (Morawski, 1926, p. 357). Ainsi, à quarante-deux ans l'homme n'est plus jeune : « On ne l'appelle plus valet » (Morawski, 1926, p. 357). Tout est mûr en août : c'est le temps de la moisson et de la rentrée des récoltes, il faut songer à amasser en prévision de la vieillesse. Parvenu à quarante-huit ans l'homme se fane comme le blé et les feuilles sur les arbres : " en ce temps yst de jeunesse / Et se change en couleur de marbre » (Morawski, 1926, p. 357). Le mois de septembre est une saison riche et féconde, mais l'homme vieillit. À cinquante-quatre ans il doit se hâter d'engranger et de vendanger pour remplir ses greniers. La soixantième année arrive avec le mois d'octobre. Cette fois, c'est la vieillesse, et il faut penser à la mort. C'est alors qu'il est bon d'être riche, sinon il faudra regretter le temps qu'on a « mal despensé » (Morawski, 1926, p. 358). À soixante-six ans, on touche au triste mois de novembre quand « [t] oute verdeur se meurt et seche » (Morawski, 1926, p. 358). L'homme qui compte soixante-six ans se rend compte que ses héritiers n'attendent que sa mort, surtout s'il est riche. En décembre, «se meurt le temps » (Morawski, 1926, p. 358), l'homme a atteint soixantedouze ans et sa vie est aussi morne que le paysage hivernal. Un lit chaud et une assiette pleine sont désormais les seuls plaisirs qui lui restent : il n'a plus qu'à mourir.

«Ainsi ne vit li homs qu'un an » (Morawski, 1926, p. 359), conclut l'auteur anonyme dans l'épilogue mettant l'accent sur la brièveté de l'existence humaine. Mais si la vie de l'homme n'est pas longue, il s'avère très vite qu'en réalité elle dure beaucoup moins que soixante-douze ans accordés par l'auteur des Douze mois figurez. D'après ses calculs, le sommeil prend à l'homme trente-six ans, c'est-à-dire la moitié de sa vie ; les quinze premières années sont vécues dans l'inconscience de l'enfance et cinq années suivantes sont enlevées par la maladie ou par l'emprisonnement. En fin de comptes, l'homme ne vit réellement que seize ans pendant lesquels il peut jouir pleinement de sa vie. Même si, comme le signale Morawski, il y a dans ces calculs une erreur qui consiste à compter doublement le temps consacré au sommeil pendant l'enfance et la maladie (Morawski, 1926, p. 352), la conclusion est d'un pessimisme saisissant qui ne fait que confirmer la tonalité amère du poème.

Ce qui frappe dans ce calcul, sauf la proportion de temps accordée au sommeil, c'est l'attitude envers l'enfance et l'adolescence, considérées comme des étapes inutiles dans la vie. Si dans les deux ouvrages analysés, l'enfance se manifeste comme 
une période pleine d'incertitude, notons pourtant un déplacement d'accent qui nous semble significatif. Alors que dans l'œuvre de Philippe de Novare l'enfance est associée au printemps, période de croissance et d'espoir, dans Les Douze mois figurez le début de la vie humaine est assimilé catégoriquement à la stérilité et au marasme caractérisant les mois hivernaux de janvier et février. «On ne peut pas s'empêcher de voir transparaître dans ce nouveau mode de représentation un regard délibérément plus étranger à l'enfance, âge froid et stérile », remarque à ce sujet Danièle Alexandre-Bidon (Alexandre-Bidon, 2003, p. 161). La petite enfance est associée au mois de janvier dont elle partage l'assoupissement et l'inertie, les six années suivantes sont assimilées à février, mois froid et peu ensoleillé, pareil en ceci à l'âge de l'apprentissage et de la dure scolarité. Et pourtant les douze ans dans l'Occident médiéval était un moment clef dans la vie de l'enfant. C'est l'âge symbolique, celui de la première manifestation publique du Christ, dont le récit se trouve dans l'Évangile de Luc : Jésus se sépare de ses parents pour discuter avec les docteurs (Lc 2, 41-52). À douze ans s'établit une sorte de prémajorité, signalé dans le texte par le début du printemps. Mais, dans l'épilogue du poème, les quinze premières années de la vie humaine sont considérées sans équivoque comme du temps perdu, celui de l'ignorance et de l'inconscience.

Au Moyen Âge, comme nous l'apprennent les travaux des historiens, la jeunesse a des contours élastiques (Lorcin, 1994, p. 16-17). Dans le traité de Philippe de Novare elle finit à quarante ans, dans Les Douze mois figurez, elle semble se prolonger d'avantage, s'éterniser même. Encore l'homme de vingt-quatre ans est appelé « enfes » et même s'il est dit qu'à trente-six ans il n'est plus « valet », ce n'est qu'à quarante-huit ans qu'il «yst de jeunesse» (Morawski, 1926, p. 357). Malgré ses atouts et attraits, la jeunesse est loin d'être un temps de jouissances et de joie de vie. Au contraire, elle apparaît comme une période d'instabilité et de dépendance, belle mais dangereuse, où les récoltes sont toujours incertaines et l'avenir n'est jamais prometteur.

Aussi bien pour Philippe de Novare que pour l'auteur des Douze mois figurez, la maturité est une période relativement courte par rapport au reste de la vie. Dans le traité des Quatre âges de l'homme, l'âge mûr est pourtant associé à l'automne, temps d'opulence et de jouissance tranquille. Par contre, dans Les Douze mois figurez, la maturité est tellement éphémère, elle semble tellement illusoire, qu'on a l'impression de ne pouvoir jamais l'atteindre. À peine sorti de la jeunesse, l'homme sent déjà le poids de la vieillesse.

La juxtaposition de deux textes: Les Quatre âges de l'homme de Philippe de Novare et Les Douze mois figurez, révèle un contraste entre le temps mesuré - objectif et froid - et la durée qui est un temps vécu, temps de l'expérience subjective qui ne se soumet pas au calcul. Comme le rappelle Agostino Paravicini Bagliani, le schéma de douze âges qui présuppose le plus grand nombre de subdivisions de la vie humaine, démontre, paradoxalement, la supériorité des saisons sur les mois (Paravicini Bagliani, 1999, p. 15). Lorsque la succession biologique est fragmentée à l'extrême, le temps du déclin apparaît comme long et inexorable. Dans le traité de Philippe de Novare, la 
comparaison des âges de la vie aux saisons de l'année permettrait d'inscrire l'existence humaine dans un temps cyclique, agricole, celui de la nature et de tout l'univers. Pour l'homme d'autrefois, comme l'a bien remarqué Philippe Ariès, cette conception de la vie pouvait être considérée comme une source de réconfort voire d'espoir : la continuité inévitable, cyclique, parfois humoristique ou mélancolique des âges de la vie était « inscrite dans l'ordre général et abstrait des choses " (Ariès, 1973, p. 40). Même si, dans l'expérience réelle, peu d'hommes avaient le privilège de parcourir tous les âges, pour Philippe de Novare qui parle en moraliste, suivre le rythme des saisons permet d'enseigner comment passer les étapes de l'existence pour ne pas la gâcher : « ausis est il des homes et des fames » (Philippe de Novare, 1888, p. 69). Dans le poème des Douze mois figurez, la mesure du temps de plus en plus comptée fait apparaître le temps individuel : une durée personnelle et subjective dont le sens échappe à l'homme (Le Goff, 1999, p. 417). À la fin du Moyen Âge on est très loin de la conception de l'homme-microcosme prônée par Hildegarde de Bingen. Non seulement l'homme n'est plus le reflet de l'univers, mais il ne s'engage même pas à lutter contre la nature, se résignant à son sort. 


\section{BIBLIOGRAPHIE}

Alexandre-Bidon, D. (2003). Seconde enfance et jeunesse dans la théorie des « âges de la vie » et le vécu familial (XIII ${ }^{\mathrm{e}}$ - début XVI ${ }^{\mathrm{e}}$ siècles). In J.-P. Bardet, J.-N. Luc, I. Robin-Romero \& C. Rollet (Eds.), Lorsque l'enfant grandit. Entre dépendance et autonomie (pp. 159-172). Paris : Presses de l'Université de Paris-Sorbonne.

Ariès, Ph. (1973). L'Enfant et la vie familiale sous l'Ancien Régime. Paris : Éditions du Seuil.

Aristote (1846). Psychologie d'Aristote. Traité de l'âme (Trans. J. Barthélemy-Saint-Hilaire). Paris : Librairie Philosophique de Ladrange.

Aristote (1883). La Rhétorique. In Aristote, Poétique et Rhétorique (Trans. Ch.-É. Ruelle) (pp. 75-336). Paris : Garnier Frères.

Batany, J. (1973). Regards sur l'enfance dans la littérature moralisante. Annales de démographie historique. Numéro thématique Enfant et Sociétés, 123-127.

Boer, C. de (Ed.) (1938). Ovide moralisé. Poème du commencement du quatorzième siècle. T. V (Livres XIV-XV). Amsterdam: J. Müller.

Cropp, G.M. (1980). Les douze mois figurez : un manuscrit et une traduction. Romania, 402, 262-271.

Dal, E. (1980). The Ages of Man and the Months of the Year. Poetry, Prose and Pictures Outlining the "Douze mois figurés" Motif Mainly found in Shepherds' Calendars and in Livres d'heures (14th to 17th Century). København: Munksgaard.

Diogène de Laërte (1847). Vies et doctrines des philosophes de l'Antiquité. T. 1 (Trans. Ch. Zevort). Paris : Charpentier.

Docampo, J. (2017). De los trabajos de los meses a los sacrificios de la virtud: el calendario del Libro de Horas Vitr/24/3 de la Biblioteca Nacional de España y sus fuentes iconográficas. Codex Aquilarensis, 33, 201-223. Retrieved from https://www.romanicodigital.com/sites/default/files/2019-09/ C33-11_Javier\%20Docampo.pdf.

Eustache Deschamps (1878). Double lay de la fragilité humaine. In Eustache Deschamps, Euvres complètes. T. 2. A.-H.-É. Queux de Saint-Hilaire (Ed.) (pp. 237-305). Paris : Firmin-Didot.

Grossel, M.-G. (2016). Le « moi » dans les Mémoires de Philippe de Novare. In Miscellanea Juslittera 1, 17-37. Retrieved from http://www.juslittera.com/wa_files/Philippe_20de_20Novare_20pdf_ 20simple.pdf.

Hildegarde de Bingen (1882). Liber divinorum operum simplicis hominis. In J.-P. Migne (Ed.), Patrologia latina. T. 197 (col. 739-1038). Paris : Migne.

Hildegarde de Bingen (1989). Le Livre des æeuvres divines (Trans. B. Gorceix,). Paris : Albin Michel.

Hüe, D. (1987). L'image de l'âge, traités et poèmes des Âges de l'homme. In Vieillesse et vieillissement au Moyen Âge (pp. 133-150). Aix-en-Provence : Presses universitaires de Provence. Retrieved from https://books.openedition.org/pup/3247.

Le Goff, J. (1999). L’Occident médiéval et le temps. In J. Le Goff, Un Autre Moyen Âge (pp. 401-420). Paris : Gallimard.

Lorcin, M.-Th. (1994). La relève des générations : sociologie, mythes et réalités. Revista d'Historia Medieval, 5, 13-40.

Mâle, É. (1910). L'Art religieux du XIII' siècle en France. Paris : Armand Colin.

Montaigne, M. de. (1962). Essais. In M. de Montaigne, Euvres complètes (Eds. A. Thibaudet \& M. Rat) (pp. 3-1097). Paris : Gallimard.

Morawski, J. (1926). Les douze mois figurez. Archivum romanicum, 10, 351-363.

Nicoud, M. (1998). Diététique et saisons. In Cl. Thomasset \& J. Ducos (Eds.), Le Temps qu'il fait au Moyen Âge. Phénomènes atmosphériques dans la littérature, la pensée scientifique et religieuse (pp. 59-68). Paris : Presses de l’Université de Paris-Sorbonne. 
Ovide (1869). Les Métamorphoses (Trans. L. Puget, Th. Guiard, Chevriau \& Fouquier). In Ovide, Euvres complètes (Ed. D. Nisard) (pp. 251-540). Paris : Firmin Didot Frères, Fils et $\mathrm{C}^{\mathrm{ie}}$.

Paravicini Bagliani, A. (1999). Âges de la vie (Trans. L. Hersant). In J. Le Goff \& J.-Cl. Schmitt (Eds.), Dictionnaire raisonné de l'Occident médiéval (pp. 7-19). Paris : Fayard.

Philippe de Novare (1888). Les Quatre âges de l'homme (Ed. M. de Fréville). Paris : Firmin Didot.

Schmitt, J.-Cl. (2007). L'invention de l'anniversaire. Annales. Histoire, Sciences Sociales, 4, 793-835.

Shakespeare, W. (1863). Comme il vous plaira. In W. Shakespeare, Euvres complètes (Trans. F. Guizot). T. 4 (pp. 213-308). Paris : Lavigne.

Tavoillot, P.-H. (2010). Le retour des âges de la vie. Le Télémaque, 37 (1), 7-10. 